\title{
Disorders Of The Basal Ganglia at the University Teaching Hospital Lusaka
}

\author{
Masharip Atadzhanov ${ }^{1}$ and Peter Mwaba ${ }^{2}$ \\ ${ }^{1}$ Department of Medicine \\ University of Zambia School of Medicine and ${ }^{2}$ University Teaching Hospital \\ Lusaka, Zambia
}

\begin{abstract}
Objective: To determine the frequency of movement disorders among adult ethnic Zambians referred to the University Teaching Hospital (UTH) Lusaka, Zambia and

logged as patients with primary neurological disorders.

Background: The Human Genome Project data showed that humans are identical across $99.9 \%$ of their genome. There is considerable evidence that despite being genetically identical, race and ethnicity appears to be an important factor in the prevalence and clinical characteristics of many, if not most, disorders. Literature review clearly shows that ethnicity has an essential role in the phenotypic expression of many movement disorders and their prevalence appears to be lowest amongst Africans.
\end{abstract}

Patients and setting: Patients attending the neurology clinic and admitted to the UTH with various neurological disorders for 5 years (January 1999-January 2004) were analysed. We evaluated patients whose symptoms met the appropriate diagnostic criteria for movement disorders and experienced symptoms at least for three days.

Results: Records were available for 4654 patients with various neurological disorders attending neurological clinic.

Of the total number of patients seen, $163(3.5 \%)$ satisfied the criteria for movement disorders. The

Corresponding Author:

Masharip Atadzhanov

Department of Medicine

University of Zambia, School of Medicine

Lusaka, Zambia most frequent syndromes of the basal ganglia were: Parkinson's disease $(31 \%)$, tremor $(24 \%)$, chorea $(20 \%)$, and dystonia (16.5\%). Myoclonus, tic, tardive dyskinesia, and other movement disorders $(8.5 \%)$ were rare in adult Zambian patients. In 25 patients $(11.3 \%)$ akinetic-rigid syndromes and hyperkinetic movement disorders were manifestations of HIV/AIDS.

Conclusions: Among patients referred for neurological services at Zambia's tertiary care teaching facility, movement disorders are relatively rare. Our study may seem to suggest that Parkinson's disease is one of the commonest movement disorders though further studies are needed to explore the role of genetics and ethnicity in the prevalence of these disorders. The presence of HIV/AIDS and its contribution to movement disorders needs to be studied further.

\section{INTRODUCTION}

S.A.K.Wilson introduced the term the extrapyramidal motor system which means the motor structures of the basal ganglia and certain related thalamic and brainstem nuclei.

Pathophysiology and clinical symptoms of the extrapyramidal motor system remains the most complex and unclear field (i.e. "terra incognita") of clinical and experimental neurology. Only a simplified version of anatomy, physiology and pathology of the extrapyramidal system considered in current textbooks ${ }^{1,2,3,4}$ are provided below.

The organization of movement is complex and exerts by 3 major systems: 1) pyramidal system 
(corticospinal and corticobulbar tracts), 2) the cerebellar system, and 3) the extrapyramidal system (EPS). Pyramidal system provides voluntary movements, muscle tone and power, the cerebellar system provides coordination of movements and muscle tone.

When we say the EPS we mean not only a group of nuclear masses in the forebrain but also related structures in the brainstem as well as neural network of connections with other parts of the nervous system.

The main structures of the EPS are: the premotor cortex, the caudate nucleus and putamen (striatum), the globus pallidum, the thalamus, the subthalamic nucleus, the nucleus ruber, the nucleus tegmenti pedunculo pontis, the substantia nigra. The principal pathways of the EPS are: the corticostriatopallidorubrospinal tract, the corticostriatopallidonigrotegmentoreticulospinal tract, olivospinal tract, and the vestibulospinal tract. These pathways run parallel to the corticospinal (pyramidal) ones; hence they were referred to as extrapyramidal, however, these nonpyramidal linkages are structurally independent. The "feedback "connections (circuits) of the EPS are:

a) striatal-pallidal-thalamic-striatal circle (loop);

b) cortical-pons-cerebellum-thalamic-cortical circle (loop);

c) thalamic-striatal-pallidal-thalamic circle (loop);

d) cortical-striatal-pallidal-nigral-thalamic-cortical circle, and

e) cortical-striatal-pallidal-thalamic-cortical circle (loop).

The EPS participates in the following physiologic functions: distribution of the muscle tone; plasticity of movements (the melody of kinesia); regulation of pose and gait; Physiologic synkinesia; automatic movements; emotional movements and expressiveness; regulation of sleep-wake cycle, the regulation of mood, Neurotransmitters of the basal ganglia. The maintenance of normal movement by the basal ganglia nuclei is ensured by a remarkable coordination between excitatory and inhibitory neurochemical signals that travel through the pathways connecting these nuclei. In the basal ganglia, there are more than 20 neurotransmitters. The most important neurotransmitter substances from the point of view of the basal ganglionic function are: glutamate, gamma-aminobutiric acid (GABA), dopamine, acetylcholine and serotonin. Less important and clearly understood neurotransmitters are neuropeptides (substance P \& encephalins), norepinephrine. Neurotransmitters exert inhibitory or excitatory effect. Acetylcholine and glutamate exert only excitatory effect, GABA only inhibitory effect, dopamine, serotonin, norepinephrine and neuropeptides have both inhibitory and excitatory effects. The cortex has direct connections with the striatum and the thalamus. The cortex sends excitatory glutamatergic fibers to the striatum. Nigrostriatal projections are dopaminergic. Degeneration of the dopaminergic Nigrostriatal neurons the pars compacta of the substantia nigra plays the key role in the pathogenesis of Parkinson's disease (PD). Degeneration of the GABAergic striatal-pallidalnigral neurons plays the key role in the pathogenesis of Huntington's disease (HD), Clinical examination of the patient with extrapyramidal disorders includes: active and passive movements, muscle tone, posture: trunk posture, stance, postural reflexes; Walking: initiation, stepping, speed; special maneuvers, and identification of involuntary movements (e.g. chorea, dystonia, tremor, and myoclonus).

\section{Symptoms of Basal Ganglia Disease}

Understanding and diagnosing of the basal ganglia diseases and disorders demands special training and long term clinical experience.

To provide a basis for understanding clinical manifestations of the basal ganglia disorders we used the symptomatic approach 5,6,7. Main clinical symptoms of the basal ganglia can be subdivided into 3 categories: bradykinesia or akinesia; rigidity and involuntary movements.

Bradykinesia (hypokinesia, akinesia) expressed by slowness in the initiation and performance of a voluntary movement. Bradykinesia may be caused by any process or drug that interrupts one or more above indicated circles in the basal ganglia.

Rigidity. Rigidity is the extrapyramidal hypertension of muscle tone (the muscles are continuously or intermittently firm and tense). 


\section{Involuntary movements (hyperkinesias, dyskinesias).}

There are 5 classic disorders described: tremor, chorea, dystonia, myoclonus, and ticks.

Tremor is by far the most commonly observed movement disorder and occurs from time to time in most normal individuals. Tremor is defined as an involuntary, rhythmic oscillation of a body part produced by synchronized contraction of antagonist muscles. Different types of tremors classified as either physiological or pathological have been described $^{8,9,10,11,12}$.

Dystonia is a disorder of abnormal posture and movement characterized by sustained muscle contraction that causes torsional and repetitive movements and abnormal postures. Dystonia is used to describe a specific type of focal or regional abnormal movement or posture, a syndrome secondary to a large number of nervous system diseases, or primary disorder when no cause can be identified. Dystonic movements may be slow or rapid and are typically repetitive by patterned, and not associated with twisting changes in posture. Rapid dystonic movements sometimes are difficult to distinguish from myoclonus, and the term 'myoclonus dystonia' is used.

Dystonia can be classified according to etiology, distribution, severity, clinical characteristics, and age at onset ${ }^{12,13,1,1,15}$.

Chorea means 'dance'. Chorea is an involuntary movement characterized by irregular, purposeless brief, jerky movements that flit from one part of the body to another in a random sequence. The character, location, and duration of individual movements are unpredictable. Chorea can occur at rest or with holding a posture. When it involves the large muscles of the trunk, shoulders, or hips, it can give a dancing quality to the movements, and when it involves the fingers it may look like typing or pianoplaying. Involuntary movements can be quick and jerky, or prolonged. If the movements involve large muscles it can cause 'ballism'. If they combined with the slower twisting movements, they are called 'choreoathetosis.' Choreoathetosis is most common in children as a form of cerebral palsy ${ }^{12,16}$.
Chorea may be the predominant manifestation of hepatolenticular degeneration, hepato-cerebral dystrophy. Chorea may be focal, asymmetric, unilateral, accompanied by Parkinsonism, ticks, or dystonia.

The causes of chorea are many. Common causes: hereditary disorders of the nervous system, druginduced disorders, systemic illnesses, toxins, metabolic disorders, and others (chorea pregnancy, senile chorea, cerebral palsy).

Myoclonus is sudden, brief, jerky, shock like involuntary movements arising from the CNS and involving extremities, face and trunk. It is one of the most commonly encountered involuntary movements ${ }^{12}$. Myoclonus can be caused by sudden muscle contraction (positive myoclonus) or by sudden muscle relaxation (negative myoclonus).

Myoclonus can happen at rest, with action, or in response to a stimulus. It can occur in single jerks, or almost continuously leading to myoclonus tremor ${ }^{17}$, 18, 19. The amplitude may range from a small contraction to a very large jerk that moves the entire body the distribution ranges from focal involvement of one body part, to segmental (involving two or more contiguous regions) to multifocal, to generalized. When the jerks occur bilaterally, they may be symmetrical or asymmetrical. Myoclonus is usually arrhythmic and irregular, but in some patients it is very regular (rhythmic) myoclonic jerks may occur spontaneously or initiated by movement or even by the intention to move. Sudden noise, light, visual threat, pinprick or touch can stimulate myoclonic jerks ${ }^{20}$.

Myoclonus is subdivided into cortical, subcortical, and spinal. Common causes of myoclonus includes, anxiety, hiccup and hereditary where no specific cause has been demonstrated.

Tics are repeated stereotyped involuntary movements or sounds. Tics are the most varied of all movement disorders. Patients demonstrate motor or vocal tics as well as wide variety of associated symptoms. Motor and vocal tics can be subdivided as 'simple' or 'complex'. Tics are usually abrupt, transient, stereotypical, coordinated movements which vary in intensity. The movements are most 
often brief and jerky (clonic), can be slower and more prolonged (tonic or dystonic tics) ${ }^{12,21,22}$. Motor tics most commonly appear as brief high velocity jerking movements of individual muscles or group of muscles, or part of the body. Common examples of simple motor tics include: eye blinking, blepharospasm, facial grimacing, head jerking, shoulder shrugging, limb jerking, abdominal or pelvic contractions, coughing, and nose wrinkling. Complex motor tics: hitting or touching parts of the body, making stereotyped sequences of movements, such as repetitive neck or limb shaking or stretching, jumping, skipping, kicking, rubbing and spitting.

\section{Less common symptoms and syndromes of the Basal Ganglia:}

Ballismus, paroxysmal dyskinesias, tardive dyskinesia, movement disorders in sleep stiff-man syndrome.

Ballism means 'to throw'. Ballism consists of throwing or flinging movements of the limbs that are usually high in amplitude and velocity and involve proximal more than distal muscles. Ballistic movements are large proximal rotatory throwing or kicking movements. Movements are irregular and unpredictable. In most cases, ballism is limited to one side of the body and is called hemiballism. If only one limb is involved-monoballism, if both legs paraballism, both sides-biballism ${ }^{12,}{ }^{23}$. The most common causes of ballism are hemorrhage or infarction in the subthalamic nucleus. ballism can be caused by pathologic process in this region: stroke, neoplasm, inflammation, trauma, metabolic causes of ballism: hyper (hypo) glycaemia, drugs: anticonvulsants, oral contraceptives, levodopa, ibuprofen.

Tardive dyskinesia (TD). TD is a clinically diverse syndrome in which choreatic, athetoid, dystonic, or other abnormal involuntary movements manifest late in the course of neuroleptic treatment. The most common signs involve orofacial and lingual movements, perioral tremor, dystal movementschoreoathetosis dystonia may accompany stereotypic or choreoathetoid movements. Tardive dyskinesia and other Involuntary movements also may be caused by a number of commonly used drugs: Dopamine agonists, anticholinergics, anticonvulsants, antihistamines, use or withdrawal of alcohol, baclofen, caffeine, calcium channel blockers, cyclosporine, digoxin, antidepressants, and so on ${ }^{24,25}$.

\section{Patients and Setting}

A study was performed to assess the prevalence of the basal ganglia diseases and symptoms among neurological in-patients and out-patients at the University Teaching Hospital.

This was a descriptive study conducted in the context of the usual clinical care according to an internal protocol. In the study were included only ethnic black adult Zambians. Informed consent was obtained from patients for video record. Clinical evaluation included a history of present illness, family history, medical history, and a review of systems with an emphasis on movement disorders, psychiatric illnesses, and cognitive function. The evaluation of movement disorders consisted of the abnormal involuntary movement scale and the unified Parkinson's disease, Huntington's disease rating scale. Most patients were examined while seated, standing and walking. Patients were also asked to speak briefly. When communication was not fully understood in English, a translator was used. Standard laboratory and radiological investigations were performed.

\section{RESULTS}

A total 4654 patients with various neurologic disorders attending neurologic clinic and admitted to University Teaching Hospital were examined between January 1999 and January 2004 (5 years). In $163(3.5 \%)$ patients' extrapyramidal symptoms were dominant clinical manifestations of neurologic illness. 163 patients with disorders of the basal ganglia included to the analysis. Frequency of the basal ganglia disorders summarized in table 1

\section{Table 1. Frequency of the basal ganglia disorders}

\begin{tabular}{|c|c|c|}
\hline Syndromes & $\mathrm{n}$ & $\%$ \\
\hline & Total & 163 \\
\hline Parkinson's disease & 51 & 31.3 \\
\hline Tremor & 39 & 24 \\
\hline Chorea & 31 & 19 \\
\hline Dystonia & 27 & 16.5 \\
\hline Myoclonus & 7 & 4.3 \\
\hline Tic & 6 & 3.7 \\
\hline Tardive dyskinesia & 2 & 1.2 \\
\hline
\end{tabular}


Of 163 patients with basal ganglia disorders most frequent was Parkinson's disease (PD) (31.3\%) (Table 2).

Table 2. Characteristics of Parkinson's disease.

$\begin{array}{lccc}\text { Sex } & \text { number } & \text { mean age } & \text { range } \\ \text { Male } & 32 & 62.8 & 36-81 \\ \text { Female } & 19 & 50.2 & 17-70 \\ \text { Familial } & 9 & 37.6 & \\ \text { Sporadic } & 42 & 54.1 & \end{array}$

PD is more frequently in male and their mean age is elder than female patients Tremor was the next frequent pathology of the basal ganglia (table 3 ).

Table 3 Main characteristics of tremor.
Table 4. Main characteristics of chorea

\begin{tabular}{lcccc} 
Disorders & \multicolumn{2}{c}{$\operatorname{sex}(\mathrm{n})$} & \multicolumn{2}{c}{ mean age } \\
& Male female & male & female \\
\hline Huntington's disease & 3 & 5 & 38.4 & 57.1 \\
Wilson's disease & 4 & - & 28.2 & - \\
Sydenham's chorea & 3 & 3 & 17.1 & 16.2 \\
Chorea gravidarum & - & 4 & - & 26.5 \\
Generalized chorea & 3 & 2 & 21.3 & 22.5 \\
Hemichorea & 2 & 2 & 37.6 & 43.4
\end{tabular}

\begin{tabular}{|c|c|c|c|c|c|c|c|c|c|c|}
\hline & Yean age & range & total, $\mathrm{n}$ & $\mathrm{S}$ & $\mathrm{y}$ & d & o & $\mathrm{m}$ & $\mathrm{e}$ & $\mathrm{s}$ \\
\hline & & & & $\begin{array}{c}\mathrm{ET} \\
\mathrm{n}\end{array}$ & $\begin{array}{c}\text { alcoh } \\
\mathrm{n}\end{array}$ & $\begin{array}{c}\text { psych } \\
\text { n }\end{array}$ & $\begin{array}{c}\mathrm{pt} \\
\mathrm{n}\end{array}$ & $\begin{array}{c}\mathrm{cb} \\
\mathrm{n}\end{array}$ & & $\begin{array}{l}\mathrm{dt} \\
\mathrm{n}\end{array}$ \\
\hline Male & 39.7 & $27-55$ & 28 & 14 & 6 & 3 & 2 & 1 & & 2 \\
\hline Female & 39.1 & $22-65$ & 11 & 8 & & & 2 & 1 & & \\
\hline
\end{tabular}

-* ET-essential tremor; alcoh-alcohol tremor; psychpsychogenic tremor; pt- posttraumatic tremor; cbcerebellar tremor, and dt-dystonic tremor.
Table 5. Main characteristics of Dystonia

\begin{tabular}{lc} 
Disorder & $\mathrm{n}$ \\
\hline Parkinsonism & 8 \\
Tremor & 6 \\
Dystonia & 5 \\
Chorea & \\
Myoclonus & 4 \\
\end{tabular}


Table 6. Distribution of patients with HIV infection.

$\begin{array}{lc}\text { Disorder } & \mathrm{n} \\ \text { Parkinsonism } & 8 \\ \text { Tremor } & 6 \\ \text { Dystonia } & 5 \\ \text { Chorea } & \\ \text { Myoclonus } & 4 \\ \end{array}$

\section{DISCUSSION}

Surprisingly, basal ganglia or extra pyramidal diseases are not as commonly seen as they are in the developed world. The reasons for this are difficult to explain but as the study was done in the hospital setting, it is difficult generalize the observations.

However, the study has noted that among the Zambian adult patients with basal ganglia disorders, the commonly seen disorders include PD, tremor, chorea and dystonia.

The clinical manifestations of basal ganglia disorders in the study patients was consistent with those observed elsewhere and reported in the literature. There are several data of interest in this study. PD is more frequent in males than female with the mean age of male patients with PD being older than female patients. Age at onset of patients with familial PD is younger than patients with sporadic PD. The course of the disease is slowly progressive and benign. The patients with PD are high responsive to levodopa treatment.

In our Zambian patients, we also noted that there is a high prevalence of patients with essential tremor and this is consistent with literature data. The course of ET in most cases is not progressive and the patients need not use antitremor drugs.

The frequent forms of chorea commonly seen in our population are: Huntington's chorea, chorea associated with pregnancy and Sydenham's chorea. We noted that most of our patients are responsive to anti-dopamine drugs and cholinoblockers. In some patients chorea is a complication of malaria and antimalaria drugs. To what extent this problem is real will need a more extensive follow up of patients on common anti-malarial drugs or pharmacovigilance.

Dystonia was observed to be more frequent in females with the mean age of onset being younger than in male. In both sexes, cranial dystonia was more common. Dystonia in our patients usually is mild in course and well responsive to dopamine antagonists.

Myoclonus, tic and tardive dyskinesia are rare in adult Zambian patients.

We have observed the following limitations of the study:

(i) This is a hospital-based study, not population-based and we recognize that the analysis was limited to participants attending the UTH inpatient and outpatient clinics

(ii) As the diagnosis was based solely on clinical grounds, misdiagnosis cannot be excluded in the absence of appropriate confirmation

(iii) As this is descriptive clinical study with no molecular genetic diagnosis, it is not possible to conclude how many patients have basal ganglia disorders due to gene mutation.

The study results provide limited evidence though that there are ethnic differences in the clinical expression of basal ganglia disorders which is consistent with the literature data ${ }^{26,27,28,29}$. It is very likely that this differences have resulted from interaction of genetic and environmental factors but more work needs to be done.

\section{ACKNOWLEDGMENT}

We thank all the patients who participated in our study and permitted us to use their clinical records. 


\section{REFERENCES}

1 Adams RD, Victor M, Ropper A.H. Principles of Neurology $7^{\text {th }}$ edition. McGraw-Hill Health Professions Division. 2003.

2 Preface to the third edition. Parkinson's disease and Movement Disorders. Eds: Jankovic J, Tolosa E, $3^{\text {rd }}$ Edition, Williams \& Wilkins, 1998.

3 Elble RJ. Motor control and movement disorders. In: Parkinson's disease and Movement Disorders. Eds, Jankovic J, Tolosa E, $3^{\text {rd }}$ edition, Williams \& Wilkins, 1998; p. $15-45$.

4 McRae A. Neurotransmitters and pharmacology of the basal ganglia. In: Parkinson's disease and Movement Disorders. Eds, Jankovic J, Tolosa E, $3^{\text {rd }}$ edition, Williams \& Wilkins, 1998; p. 47-65.

5 Marsden CD. Movement disorder symptomatology. In: Advances in Neurology 1998, p.67-86.

6 Sanger TD, Lang AE. Case studies in paediatric movement disorders. Movement Disorders, CD-ROM 2003.

7 AAN Clinical Practice Guidelines. CD-ROM 2003 edition

8 McAuley J, RothwellJ. Identification of psychogenic, dystonic, and other organic Tremors by a coherence entrainment test. Mov Disord 2004; 19:253-267.

9 Marshall V, Grosset DG. Role of dopamine transporter imaging in the diagnosis Of Atypical tremor disorders. Mov Disord 2003; 18(Suppl.7):S22-S27.

10 Deuschl G, Raethjen J, Lindermann M, Krack P. The pathophysiology of tremor. Muscle Nerve 2001; 24(6):716-735.

11 Potts F. Tremors. In: Office Practice in Neurology. Eds.Samuels MA, Feske SK. $2^{\text {nd }}$ Edition 2003, Churchill Livingstone. p. 798802.

12 Lang AE. Movement disorder symptomatology. In: Neurology in Clinical Practice $4^{\text {th }}$ edition. Ed.BradleyWG, Daroff RB, Fenichel GM, Jankovic J. Butterworth Heinemann, 2003, p. 299-320.

13 JankovicJ, Fahn S. Dystonic disorders. In: Parkinson's disease and Movement Disorders. Eds. Jankovic J, Tolosa E. $3^{\text {rd }}$ edition, Williams \& Wilkins, 1998, p. 513-552.

14 Jankovic J. Primary and secondary generalized dystonias. In: Office Practice in Neurology. Eds.Samuels MA, Feske SK. $2^{\text {nd }}$ edition 2003, Churchill Livingstone. p. 816-821.
15 Charness ME. Task-specific focal dystonia. In: Office Practice in Neurology. Eds.Samuels MA, Feske SK. $2^{\text {nd }}$ edition 2003, Churchill Livingstone. p. 827-830.

16 O'Brien CF. Chorea. In: Parkinson's disease and Movement Disorders. Eds. Jankovic J, Tolosa E. $3^{\text {rd }}$ edition, Williams \& Wilkins, 1998. p. 357-364.

17 Shibasaki H. Myoclonus and startle syndromes. In:Parkinson's disease and Movement Disorders. Eds. Jankovic J, Tolosa E. $3^{\text {rd }}$ edition, Williams \& Wilkins, 1998. p. 453-464.

18 Fahn S. Overview, history, and classification of Myoclonus. Adv Neurol 2002; 89:13-17.

19 Molloy F, Hallett M. Myoclonus. . In: Office Practice in Neurology. Eds.Samuels MA, Feske SK. $2^{\text {nd }}$ edition 2003, Churchill Livingstone. p. 834-839.

20 Rivest J. Myoclonus. Can J Neurol Sci 2003; 3 (suppl 1):S53-S58.

21 Tolosa E, Jankovic J. Tics and Tourette's syndrome. In: Parkinson's disease and Movement Disorders. Eds.Jankovic J, Tolosa E $3^{\text {rd }}$ edition, Williams \& Wilkins, 1998 p. 491-512.

22 Marshall FJ, Kurlan R. Tics and Tourette's syndrome. In: Office Practice in Neurology. Eds.Samuels MA, Feske SK. $2^{\text {nd }}$ edition 2003 , Churchill Livingstone. p. 830-834.

23 Shannon K. Ballism. In: Parkinson's disease and Movement Disorders. Eds. Jankovic J, Tolosa E. $3^{\text {rd }}$ edition, Williams \& Wilkins, 1998, p. 491-512.

24 Fernandez HH, Friedman JH. Classification and treatment of tardive syndromes. Neurologist 2003; 9(1)16:27-29.

25 Miyawaki E. Tardive dyskinesia and other drug-related movement disorders. In: Office Practice in Neurology. Eds.Samuels MA, Feske SK. $2^{\text {nd }}$ edition 2003, p. 810-815.

26 Ubramony SH, Hernandez D, Adam A, et al. Ethnic differences in the expression of neurodegenerative disease. Mov Disord 2002; 17(5):1068-1071.

27 Hu MTM, Chauhury KR, Jarosz J, et al. An imaging study of parkinsonism among African-Caribbean and Indian London communities. Mov Disord 2002; 17(6):13211328.

28 Louis ED, Barnes LF, Ford B, et al. Ethnic differences in Essential tremor. Arch Neurol 2000; 57:723-727.

29 Ragothaman M, Murgod UA, Gururaj G, et al. Lower risk of Parkinson's disease in an admixed population of European and Indian origins. Mov Disord 2003; 18(8):912-914. 